\title{
Length-based separation of Bacillus subtilis bacterial populations by viscoelastic microfluidics
}

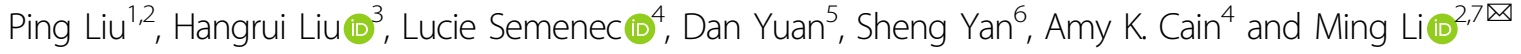

\begin{abstract}
In this study, we demonstrated the label-free continuous separation and enrichment of Bacillus subtilis populations based on length using viscoelastic microfluidics. B. subtilis, a gram-positive, rod-shaped bacterium, has been widely used as a model organism and an industrial workhorse. B. subtilis can be arranged in different morphological forms, such as single rods, chains, and clumps, which reflect differences in cell types, phases of growth, genetic variation, and changing environmental factors. The ability to prepare B. subtilis populations with a uniform length is important for basic biological studies and efficient industrial applications. Here, we systematically investigated how flow rate ratio, poly(ethylene oxide) (PEO) concentration, and channel length affected the length-based separation of $B$. subtilis cells. The lateral positions of $B$. subtilis cells with varying morphologies in a straight rectangular microchannel were found to be dependent on cell length under the co-flow of viscoelastic and Newtonian fluids. Finally, we evaluated the ability of the viscoelastic microfluidic device to separate the two groups of B. subtilis cells by length (i.e., $1-5 \mu \mathrm{m}$ and $>5 \mu \mathrm{m}$ ) in terms of extraction purity (EP), extraction yield (EY), and enrichment factor (EF) and confirmed that the device could separate heterogeneous populations of bacteria using elasto-inertial effects.
\end{abstract}

\section{Introduction}

Bacillus subtilis is a model gram-positive, rod-shaped bacterium commonly found in soil and the gastrointestinal (GI) tract of ruminants and humans. It is generally recognized as safe (GRAS) by the US Food and Drug Administration (FDA) and widely used as a cell factory for the industrial production of heterologous proteins, pharmaceuticals, and functional biomaterials due to its propensity for genetic manipulation and ease of culture $^{1}$. Given these favorable attributes, B. subtilis has served as a model organism for diverse studies on physiology, metabolism, and genetic manipulation ${ }^{2}$. B. subtilis has been intensively studied over the past decades due to its importance in the biotechnology industry and basic biological research ${ }^{3}$.

\footnotetext{
Correspondence: Ming Li (ming.li@mq.edu.au)

'Suqian University, Suqian 223800, China

${ }^{2}$ School of Engineering, Macquarie University, Sydney, NSW 2109, Australia

Full list of author information is available at the end of the article

These authors contributed equally: Ping Liu, Hangrui Liu
}

B. subtilis populations typically exhibit numerous cellular arrangements, such as single rods, chains of varying length connected by uncleaved septal wall material, sporulating cells, and biofilms comprised of matrixproducing cells. These heterogeneous cell states depend on various factors, such as the individual cell properties, the phase of growth, genetic variations, and environmental stressors ${ }^{4-6}$. Standard laboratory strains of $B$. subtilis are known to exist in two main morphologically distinct forms, and this cell population heterogeneity is related to the growth phase. Single motile cells or doublets occur more often during stationary phase transition, whereas long chains of sessile cells are more common in the exponential phase ${ }^{7,8}$. B. subtilis can alter cellular length via intrinsic genetic modifications, and mutant strains show distinct morphological traits. Two key genes, $m r e B$, and $m b l$, have been shown to play leading roles in B. subtilis cell shape determination ${ }^{9}$. Moreover, the length of $B$. subtilis can vary in response to environmental stimuli, such as temperature, antibiotic exposure, $\mathrm{pH}$, and nutrient composition ${ }^{10-12}$. 
Given the morphological and regulatory heterogeneity of B. subtilis cell cultures throughout the different growth phases and in response to certain conditions, it is advantageous to these fix cells after treatment at a predetermined time point for many experiments (e.g., those that assess the effects of exposure to environmental stimuli or stress). The fixation ensures that the cells maintain a homogenous morphological state and uniform growth phase during subsequent molecular and morphological examinations. Fixed cells after morphology-based separation can be visualized by microscopy, atomic force microscopy (AFM), or scanning electron microscopy (SEM) to assess changes to their ultrastructures under certain conditions, although selection and specific treatment of cells must be performed prior to fixation. Visualizing changes to bacterial cell morphology provides insight into their behaviors and mechanisms for dealing with environmental stresses, such as their response to antibiotics or host immune cells, that allow them to compete in microbial communities ${ }^{13}$. Furthermore, the fixed populations sorted by length can be investigated on a molecular level through DNA, RNA, and protein extraction followed by genomic, transcriptomic, or proteomic analyses to identify key molecular differences between morphologically distinct populations.

Unfortunately, traditional enrichment and separation approaches, such as centrifugal elutriation and membrane filtration, separate cells mainly by size, and they require specialized and costly equipment that also needs a substantial input size, which is not always possible with precious clinical samples. A previous technique incorporated centrifugal elutriation into a microfluidic device ${ }^{14}$ to address these issues and was able to separate polymer particles 1,3 , and $5 \mu \mathrm{m}$ in diameter; however, this system has not been used for the separation of bacterial cells. Although cells with different morphologies can be isolated using micropipettes under a light microscope $\mathrm{e}^{15}$, the process has a low throughput and is labor-intensive.

Several microfluidic techniques featuring low cost, high throughput, and high accuracy have been developed for shape-based particle and cell separation ${ }^{16}$. Some techniques are based on integration with externally applied fields, such as dielectrophoresis (DEP) ${ }^{17}$ and magnetophoresis $^{18}$, but these always require a complex fabrication process and a bulky external setup, and external actuation may cause adverse effects on the cells (e.g., cell damage by Joule heating). Other techniques rely on internal channels or microstructure-induced microflows, such as deterministic lateral displacement (DLD) ${ }^{19}$, hydrophoresis ${ }^{20}$, and inertial focusing ${ }^{21,22}$. For example, DLD has been used for the separation of Streptococcus pneumoniae based on chain length ${ }^{23}$ and shape-based separation of other different bacterial species ${ }^{24}$ and inertial microfluidics has enabled the separation of spherical and elliptical bacterial cells $^{25}$. However, the first two techniques require complex networks of microstructures (e.g., branched channels or post and groove arrays). Inertial microfluidics has been extensively studied for relatively large cells (typically > $2 \mu \mathrm{m})$, such as blood cells and circulating tumor cells $(\mathrm{CTCs})^{26-28}$. Although the manipulation of bacteria with sub-micro scale resolution has been achieved recently using inertial microfluidics, this requires either a long curved microchannel $^{29}$ or an oscillatory technique with the aid of external instruments (including pressure sources, signal generators, circuits, and valves) ${ }^{27}$. There have been no reports on the separation of a single species of bacterial cells using inertial microfluidics.

In this study, we adopted a viscoelastic microfluidics technique for the label-free continuous separation and enrichment of $B$. subtilis populations based on length. Although a co-flow of viscoelastic and Newtonian fluids can be used to separate both synthetic and biological particles ${ }^{28,30,31}$, such as the isolation of bacteria, platelets, and CTCs from whole blood ${ }^{32-35}$, the separation of Staphylococcus aureus from platelets ${ }^{36}$ and the separation of exosomes from other extracellular vesicles ${ }^{37}$, these achievements have been accomplished for two or more species and are mainly based on size. Yuan et al. recently demonstrated the shape-based separation of gramnegative Cyanobacterial anabaena cells using viscoelastic microfluidics ${ }^{38}$. Since $C$. anabaena bacterial cells are relatively large (up to $\sim 1000 \mu \mathrm{m}$ in length), they are easy to manipulate by microfluidic techniques. To date, it has not been shown whether elasto-inertial effects can be used to separate gram-positive bacterial cells based on shape in a co-flow system. Here, we examined the effects of different factors, including the flow rate ratio of the sheath and sample fluids, poly(ethylene oxide) (PEO) concentration, and channel length, on the lateral positions of B. subtilis cells of different lengths. Shorter and longer cells were found to exit mostly from the outlets closer to the channel sidewalls and center, respectively. To the best of our knowledge, this is the first report on the chain length-based separation and enrichment of a single species of gram-positive bacterial cells using viscoelastic microfluidics.

\section{Theory}

The dynamics of fluid and particle motion in viscoelastic flows are characterized by the following three dimensionless numbers: the Reynolds number, the Weissenberg number, and the elasticity number. The values of these three dimensionless numbers at different flow rates and PEO concentrations used in the experiment are listed in the Supplementary Information (see Tables S1 and S2). 
The Reynolds number, $R e$, is defined as the ratio between inertial force and viscous force ${ }^{39}$ :

$$
R e=\frac{\rho U D_{h}}{\eta}=\frac{2 \rho Q}{\eta(w+h)}
$$

where $\rho$ is the fluid density, $U$ is the average velocity, $D_{h}=2 w h /(w+h)$ is the hydraulic diameter of a rectangular channel ( $w$ and $h$ are the channel width and height, respectively), and $Q=Q_{s}+Q_{s h}$ is the volumetric flow rate $\left(Q_{s}\right.$ and $Q_{s h}$ are the sample and sheath flow rate, respectively).

The Weissenberg number, $w_{i}$, measures the fluid elasticity effects, and is defined $\mathrm{as}^{40}$ :

$$
W_{i}=\frac{\lambda}{t_{f}}=\lambda \dot{\gamma}=\lambda \frac{2 U}{w}=\frac{2 \lambda Q}{h w^{2}}
$$

where $t_{f}$ is the characteristic time, which is approximately equal to the inverse of the average shear rate $\dot{\gamma}\left(=\frac{2 U}{w}\right.$ or $\frac{2 Q}{h w^{2}}$ in a rectangular microchannel).

The elasticity number, $E l$, is defined as the ratio of $w_{i}$ to Re:

$$
E l=\frac{W_{i}}{R e}=\frac{\lambda \eta(w+h)}{\rho w^{2} h}
$$

Details of the mechanisms of particle motion dynamics under elasto-inertial effects can be found in our previous study $^{26}$. In general, a particle flowing through a rectangular microchannel in a viscoelastic fluid experiences two main forces, elastic and inertial lift forces. The elastic lift force, $F_{e l}$, is generated by the nonuniform normal stress differences. Since the magnitude of the second normal stress difference, $N_{2}\left(=\tau_{y y}-\tau_{z z}\right)$, is much smaller than that of the first normal stress difference $N_{1}\left(=\tau_{x x}-\tau_{y y}\right)$ (where $\tau_{x x}, \tau_{y y}$, and $\tau_{z z}$ are the normal stresses exerted in the flow for the velocity gradient and vorticity direction, respectively) ${ }^{41}$, the effects of $N_{2}$ can be neglected in dilute PEO solutions. Hence, the elastic lift force, $F_{e l}$, is proportional to the variation of $N_{1}$ over the size of the particle and can be expressed as:

$$
F_{e l}=C_{e l} a^{3} \nabla N_{1}=C_{e l} a^{3}\left(\nabla \tau_{x x}-\nabla \tau_{y y}\right)=-2 C_{e l} a^{3} \eta_{p} \lambda \nabla_{\gamma}^{\cdot 2}
$$

where $C_{e l}$ is the nondimensional elastic lift coefficient, a is the equivalent spherical diameter of the particle, and $\dot{\gamma}$ is the average fluid shear rate in the channel width direction.

The inertial lift force, $F_{i l}$, is composed of a wall-induced lift force, $F_{i l_{-} w}$, that pushes the particle away from the wall, and a shear gradient-induced lift force, $F_{i l \_s}$, which directs the particle from the channel center toward the walls. The net inertial lift force, $F_{i l}$, exerted on a spherical particle is given by:

$$
F_{i l}=F_{i l \_w}+F_{i l \_s}=C_{i l} \rho U^{2} a^{4} / h^{2}
$$

where $C_{i l}$ is the lift coefficient as a function of the Reynolds number and the normalized cross-sectional position.

As the elastic lift force $F_{e l}$ and the inertial lift force $F_{i l}$ scale differently with equivalent spherical particle diameters, particles with different morphologies (e.g., size and shape) can be directed to distinct equilibrium positions in the channel width direction under the combined effects of $F_{e l}$ and $F_{i l}$. Key external parameters that affect particle motion include the flow rate, channel dimensions, and effective relaxation time (PEO concentration). The co-flow of Newtonian and viscoelastic fluidics used in our study generates a stable interface between the sample and sheath flows close to the sidewall of the microchannel. This leads to wall-directed interfacial elastic lift forces that compete with center-directed inertial lift forces and influence the migration of cells from the Newtonian fluid to the viscoelastic fluid. It has been reported that the elastic lift force acting on the particles is dramatically altered at the interface due to the absence of elastic stresses at the Newtonian sample flow. For example, the elastic lift forces are directed toward the sidewall and centreline, respectively, when the far wall side of the particle is at the interface and the particle is fully immersed in the viscoelastic fluid ${ }^{36}$. A more detailed analysis of the interfacial elastic force acting on the particle in a co-flow system can be found elsewhere ${ }^{36}$.

\section{Results and discussion}

The viscoelastic microfluidic device for the length-based separation of $B$. subtilis cells used in our experiment has four main elements: two inlets, a straight rectangular microchannel (height/width $=2.5$ ), an expansion region, and seven outlets (see Fig. 1a). B. subtilis cell suspensions were injected via the side inlet, while sheath (viscoelastic non-Newtonian) fluids containing PEO were injected via the middle inlet. Due to the sheath flows, B. subtilis cells were prealigned into narrow focal streams along the channel sidewalls at the inlet. An interface of viscoelastic and Newtonian fluids formed close to the sidewall of the straight microchannel $(\sim 1.7 \mu \mathrm{m}$, see Supplementary Information Fig. S1). In the rectangular microchannel, $B$. subtilis cells with different morphologies gradually migrated toward the channel center at a length-dependent speed. Longer $B$. subtilis cells penetrated the interface and thus migrated to the channel center faster than the shorter $B$. subtilis cells that were intercepted by the interface, resulting in different lateral positions of $B$. subtilis cells that were dependent on cell length. The differences in cell length-dependent lateral positions were 


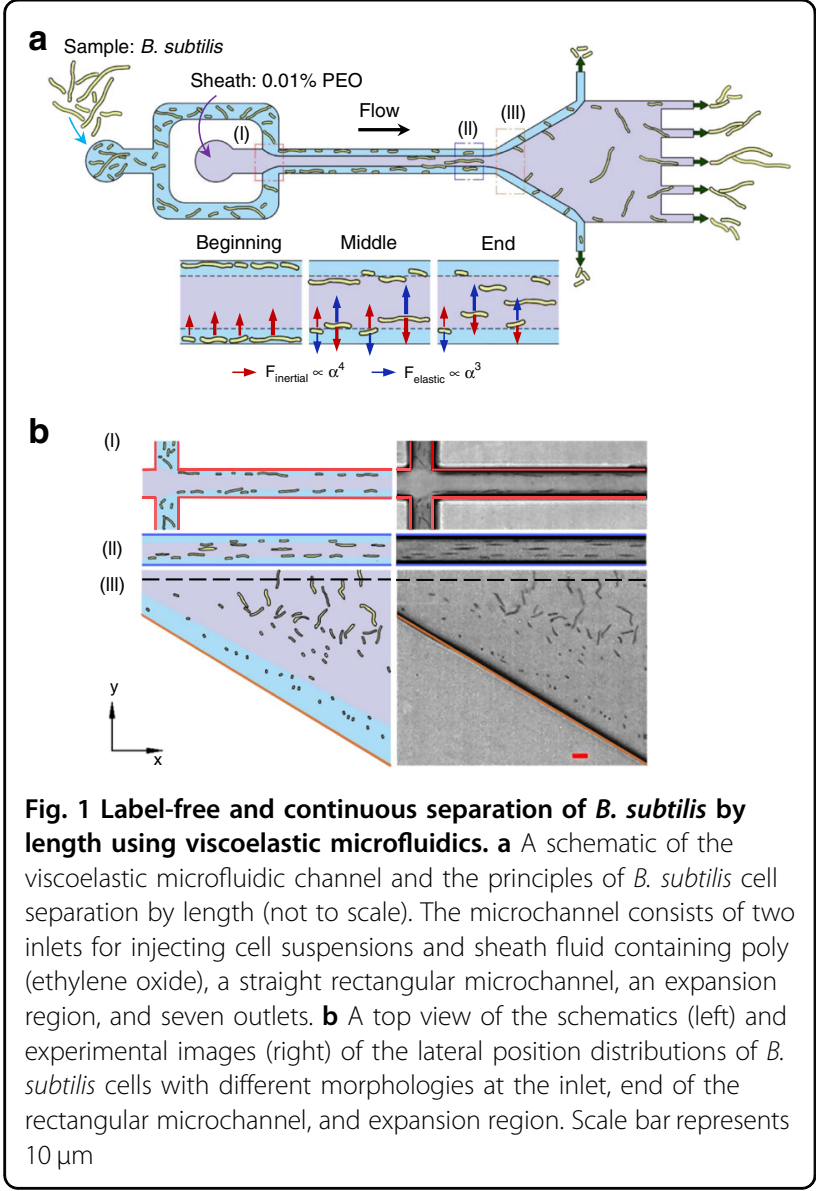

enhanced by the expansion region, and B. subtilis cells with different lengths were finally directed by different outlets: shorter and longer cells exited from the outlets close to the channel sidewalls and channel centerline, respectively. Notably, cell curvature was not found to significantly affect the lateral positions of the cells (see Fig. S2). Schematics and experimental images of the distribution of $B$. subtilis cells with different lengths at the inlet, end of the rectangular microchannel $(14.78 \mathrm{~mm}$ downstream of the inlet), and expansion region $(15.18 \mathrm{~mm}$ downstream of the inlet; channel width of $\sim 235 \mu \mathrm{m}$ ) are presented in Fig. 1b, demonstrating lengthbased separation of B. subtilis cells in the proposed viscoelastic microfluidic device.

\section{Lateral positions depend on $B$. subtilis cell length}

We examined the lateral positions of B. subtilis with different morphologies, which were found to be dependent on cell length. The B. subtilis cell suspension and $100 \mathrm{ppm}$ PEO solution were injected into the viscoelastic microfluidic device at flow rates of 5 and $40 \mu \mathrm{L} / \mathrm{min}$, respectively. The corresponding Re value was 24.91 and Wi was 9.23. These two dimensionless parameters were calculated using Eqs. (1) and (2), with a channel width of
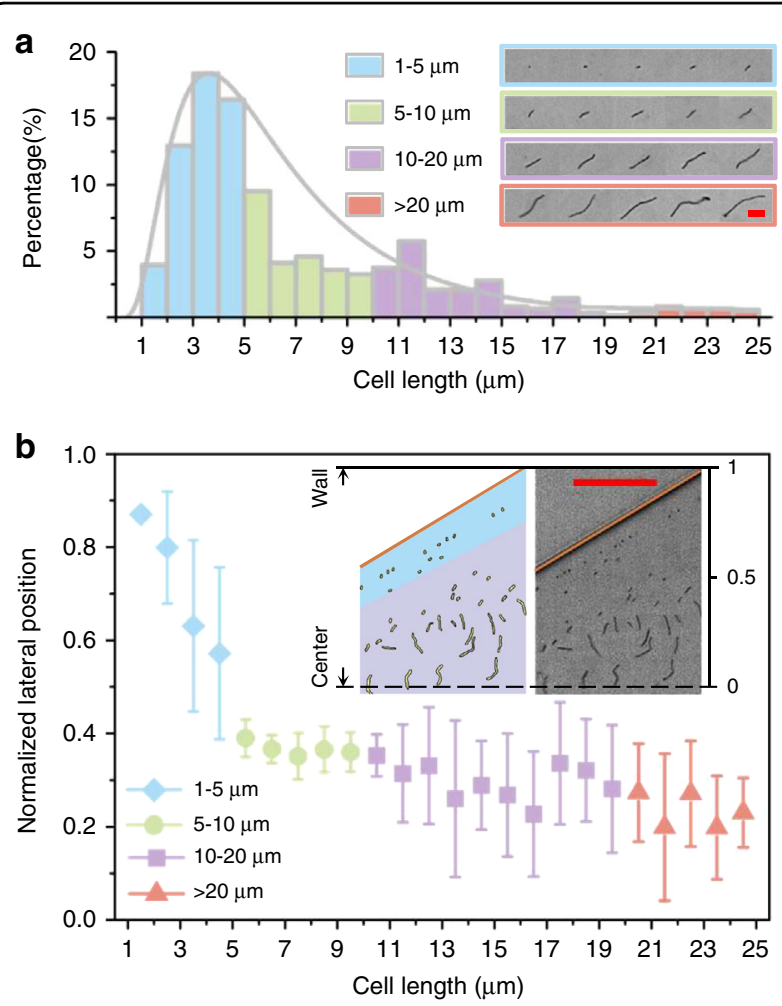

Fig. 2 The lateral positions of $B$. subtilis cells depend on cell length. a Histogram of the chain length distribution of four groups of

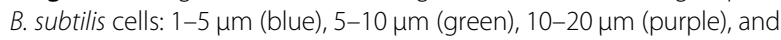
$>20 \mu \mathrm{m}$ (pink). The inserts are images showing four groups of $B$. subtilis cells with different lengths. The scale bar represents $10 \mu \mathrm{m}$. b Plot of the normalized lateral positions of the four groups of $B$. subtilis cells with different lengths. The error bars indicate the standard deviations obtained from at least 20 measurements. The insets are a schematic (left) and an experimental image (right) of the normalized lateral position distributions of $B$. subtilis cells of different lengths at the expansion region. The scale bar represents $50 \mu \mathrm{m}$

$20 \mu \mathrm{m}$ and a height of $50 \mu \mathrm{m}$ and the values of density $\rho$, dynamic viscosity $\eta$ and effective relaxation time $\lambda$ of the PEO solutions can be found in Table S3. The lengths of the $B$. subtilis cells ranged from 1.2 to $24.6 \mu \mathrm{m}$ with an approximate medium value of $6.8 \pm 0.3 \mu \mathrm{m}$, whereas the widths of the $B$. subtilis cells were all approximately $0.8 \mu \mathrm{m}$ (see Fig. 2a). We divided the B. subtilis cells into four groups based on cell length: $1-5 \mu \mathrm{m}, 5-10 \mu \mathrm{m}$, $10-20 \mu \mathrm{m}$, and $>20 \mu \mathrm{m}$, that represent $51.4 \%, 24.9 \%$, $20.5 \%$, and $3.2 \%$ of the population, respectively. Fig $2 \mathrm{~b}$ compares the normalized lateral positions of the four groups of B. subtilis cells at an expansion region $(15.2 \mathrm{~mm}$ downstream of the inlet, channel width of $\sim 260 \mu \mathrm{m})$. An expansion region rather than a $20 \mu \mathrm{m}$ wide rectangular channel region was selected for measurements to easily visualize the differences in cell lateral positions. Since two sets of lateral positions symmetric to the channel centerline were found for $B$. subtilis cells with different 
lengths, only the cell lateral positions in half of the expansion region were shown (see Fig. 2b, insets). The results showed that the normalized lateral position generally decreases with increasing $B$. subtilis cell length; longer cells were present in lateral positions closer to the channel centerline than shorter cells (see Fig. $2 b$ and Supplementary Movie S1).

Moreover, we investigated the capability of inertial microfluidics alone for the length-based separation of $B$. subtilis cells using the same device (see Supplementary Information Figure S3). The lateral positions of the longer cells were found to be closer to the channel wall, reducing the difference in lateral position for the two groups of cells (i.e., cells $<5 \mu \mathrm{m}$ and cells $>5 \mu \mathrm{m}$ ). This finding was confirmed after comparing the lateral positions of $B$. subtilis cells at the outlets (see Supplementary Information Fig. S2). A portion of longer cells $(>5 \mu \mathrm{m})$ and shorter cells $(<5 \mu \mathrm{m})$ were found to exit from the same outlet (see Fig. S4a) using inertial microfluidics alone; hence, reduced separation efficiency of these two groups based on chain length occurred. We also tested the ability of the device to separate $B$. subtilis cells of various lengths using viscoelastic microfluidics alone (see Figure S5), and no clear separation was observed due to the large overlaps in the cell lateral positions.

\section{Effects of the flow rate ratio}

We first investigated the effects of the flow rate ratio $\alpha$ $\left(=Q_{s h} / Q_{s}\right)$ on the lateral positions of $B$. subtilis cells with different morphologies. The flow rate of the sheath fluid containing $100 \mathrm{ppm}$ PEO in PBS was fixed at $40 \mu \mathrm{L} / \mathrm{min}$, while the sample fluid containing $B$. subtilis cells of various lengths was injected into a $15 \mathrm{~mm}$ long microchannel at five different flow rates, $2,3,4,5$, and $10 \mu \mathrm{L} / \mathrm{min}$. The flow rate ratios $\alpha$ were $20,13.3,10,8$, and 4 respectively. The corresponding $\mathrm{Re}, \mathrm{Wi}$, and $\mathrm{El}$ values are listed in Supplementary Information Table S1. According to Eqs. (1) and (2), both Re and Wi increased with an increase in the total flow rate (also a decrease in $\alpha$ ), which leads to enhanced inertial and elastic effects. However, El remained constant at 0.37 (see Eq. (3)), indicating that inertial lift force dominates in the entire range of $\alpha^{28}$.

When $\alpha$ decreased from $20\left(Q_{s}=2 \mu \mathrm{L} / \mathrm{min}\right)$ to $10\left(Q_{s}=\right.$ $4 \mu \mathrm{L} / \mathrm{min}$ ), the lateral positions of the four groups of $B$. subtilis cells were found to migrate slightly toward the channel wall (see Fig. 3). This is mainly a result of the slightly increased dominant inertial lift forces, which direct the long cells that traverse the interface and are located in the viscoelastic non-Newtonian fluid toward the channel sidewall. As $\alpha$ was further decreased to $8\left(Q_{s}\right.$ $=5 \mu \mathrm{L} / \mathrm{min}$ ), the three groups of longer $B$. subtilis cells (i.e., $5-10 \mu \mathrm{m}, 10-20 \mu \mathrm{m}$, and $>20 \mu \mathrm{m}$ ) appeared to migrate slightly toward the channel centerline, while the shorter B. subtilis cells (i.e., $1-5 \mu \mathrm{m}$ ) appeared to migrate
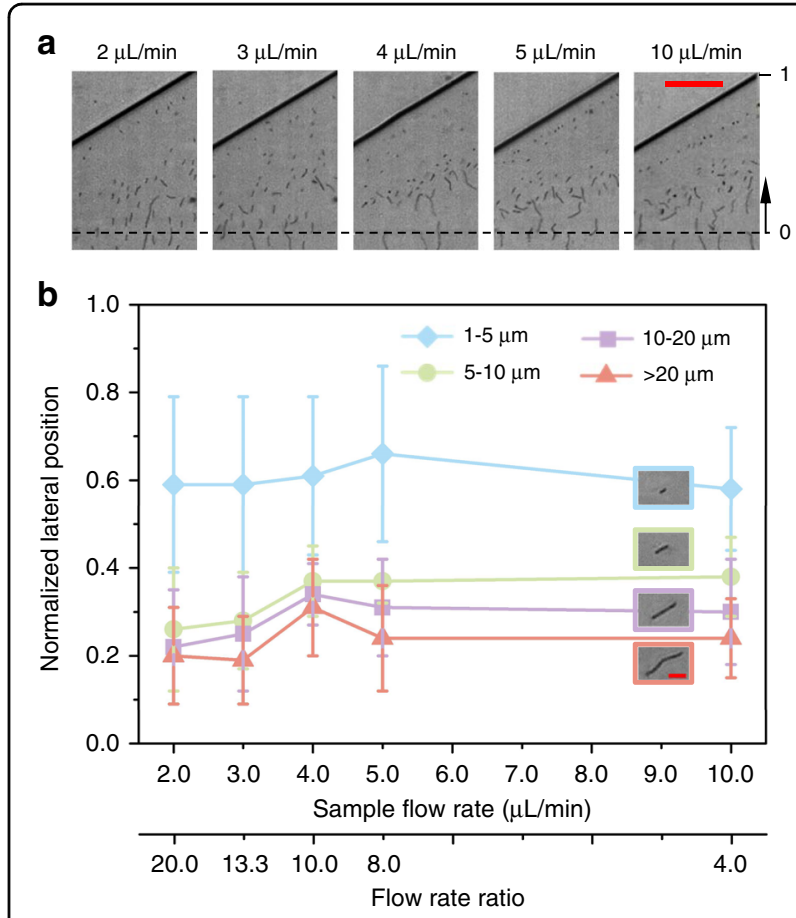

Fig. 3 Effects of flow rate ratio on the separation of $B$. subtilis cells using a $100 \mathrm{ppm}$ PEO solution in a $15 \mathrm{~mm}$ long rectangular microchannel. The sheath flow rate was fixed at $40 \mu \mathrm{L} / \mathrm{min}$, while the sample fluid varied, at 2, 3, 4, 5, and $10 \mu \mathrm{L} / \mathrm{min}$. The Re values were $23.25,23.8,24.35,24.91,27.67$, respectively, and Wi values were 8.61, $8.82,9.02,9.23,10.25$, respectively. a Experimental images of $B$. subtilis cells with various lengths at the expansion region for the five different sample flow rates. The black dashed lines represent the channel centerlines. The scale bar represents $50 \mu \mathrm{m}$. b Plots of the average normalized lateral positions for the four groups of $B$. subtilis cells with different lengths: 1-5 $\mu \mathrm{m}$ (blue), 5-10 $\mu \mathrm{m}$ (green), 10-20 $\mu \mathrm{m}$ (purple), and $>20 \mu \mathrm{m}$ (pink). The error bars indicate the standard deviations obtained from at least 100 measurements

slightly toward the channel sidewall. This resulted in an increase in the gap between the average normalized lateral positions of the four groups of $B$. subtilis cells (see Fig. 3b). This result is probably because the focusing of the cells at the beginning of the straight channel decreased, reducing the cell-to-cell interactions in the regions closer to the channel wall. Upon decreasing $\alpha$ further to $4\left(Q_{s}=10 \mu \mathrm{L} / \mathrm{min}\right)$, shorter $B$. subtilis cells (i.e., $1-5 \mu \mathrm{m})$ tended to move closer to the channel centerline, reducing the gap between the average normalized lateral positions. This is mainly because in Newtonian fluids, the increasing inertial lift forces direct shorter $B$. subtilis cells away from the wall.

We also examined how the sheath flow rate affected the migration of $B$. subtilis cells with different morphologies by increasing the sheath flow rate from 10 to $50 \mu \mathrm{L} / \mathrm{min}$ while keeping the sample flow rate constant at $5 \mu \mathrm{L} / \mathrm{min}$ (see Supplementary Information Figure S6). The results showed that there was no significant variation in the 
average normalized lateral positions of $B$. subtilis cells at different sheath flow rates (except when the shorter $B$. subtilis cells with lengths of $1-5 \mu \mathrm{m}$ appeared to migrate toward the channel wall when the sheath flow rate was $40 \mu \mathrm{L} / \mathrm{min}$ ). The length-based separation of B. subtilis cells was found to not be very sensitive to flow rates, probably because both the elastic and inertial lift forces are proportional to $U^{2}$ (see Eqs. (4) and (5)). This observation agrees with the previous study by Lu et al. ${ }^{42}$, where the lateral positions of spherical and peanut particles remained almost unchanged at different flow rates.

\section{Effects of PEO concentration}

We further investigated the effects of PEO concentration $c$ on the lateral positions of $B$. subtilis cells with different morphologies. Here, we used three different concentrations of PEO solutions, 100, 500, and $1000 \mathrm{ppm}$. The sample and sheath flows were injected into the microchannel with a length of $15 \mathrm{~mm}$ at the fixed flow rates of $5 \mu \mathrm{L} / \mathrm{min}$ and $40 \mu \mathrm{L} / \mathrm{min}$, respectively. The Re values were $24.91,21.52$, and 18.39 , respectively, and the Wi values were 9.23, 26.25, and 41.25, respectively (see Table S2).

When the PEO concentration $c$ was $100 \mathrm{ppm}(\mathrm{El}=$ 0.37 ), there was a clear difference in the lateral positions of the four groups of B. subtilis cells (see Fig. 4). A portion of short B. subtilis cells (i.e., $1-5 \mu \mathrm{m}$ ) were trapped at the interface of the viscoelastic and Newtonian fluids, while longer $B$. subtilis cells penetrated the interface and reached the lateral equilibrium positions in the viscoelastic fluid that were dependent on cell length. When $c$ increased to $500 \mathrm{ppm}(\mathrm{El}=1.22)$, the three groups of longer B. subtilis cells (i.e., 5-10 $\mu \mathrm{m}, 10-20 \mu \mathrm{m}$, and $>20 \mu \mathrm{m}$ ) tended to move closer to the channel wall, resulting in a decrease in the distance between the normalized lateral positions. This is because wall-directed elastic lift forces began to dominate the center-directed inertial lift force at the interface, and a longer cell length is required to penetrate the interface. Tian et al. ${ }^{36}$ observed that larger particles (i.e., $2 \mu \mathrm{m}$ in diameter) migrated from the centerline to the sidewalls when the PEO concentration increased from $100 \mathrm{ppm}$ to $1000 \mathrm{ppm}$.

As $c$ was further increased to $1000 \mathrm{ppm}(\mathrm{EI}=2.24)$, the elastic lift force became more dominant. A larger portion of short B. subtilis cells in the first two groups (i.e., $1-5 \mu \mathrm{m}$ and $5-10 \mu \mathrm{m})$ were trapped at the interference, resulting in a shift in the average normalized lateral position of these two groups toward the channel sidewall. However, the average normalized lateral position of $B$. subtilis cells in the other two groups (i.e., $10-20 \mu \mathrm{m}$ and $>20 \mu \mathrm{m}$ ) shifted slightly toward the microchannel centerline. The PEO concentration $c$ of the sheath fluid has complex effects on cell migration ${ }^{37}$. As the effective relaxation time $\lambda$ of the PEO concentration increases with
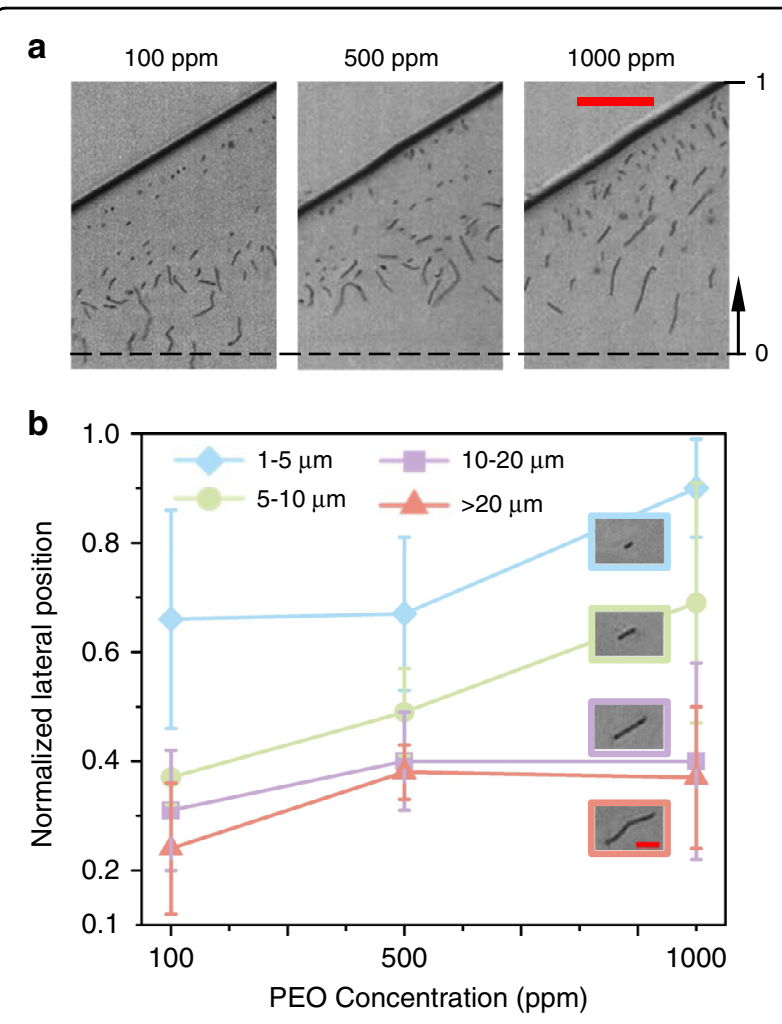

Fig. 4 Effects of PEO concentration on the separation of $B$. subtilis cells in a $15 \mathrm{~mm}$ long rectangular microchannel with three different concentrations of PEO solutions: 100,500 , and $1000 \mathrm{ppm}$. The flow rates of the sample and sheath were $5 \mu \mathrm{L} / \mathrm{min}$ and $40 \mathrm{\mu L} / \mathrm{min}$, respectively. The Re values were $24.91,21.52,18.39$, respectively, and the Wi values were $9.23,26.25,41.25$, respectively. a Experimental images of $B$. subtilis cells of various lengths at the expansion region for the three different PEO concentrations. The black dashed lines represent the channel centerlines. The scale bar represents $50 \mu \mathrm{m}$. b Plots of the average normalized lateral positions for four groups of $B$. subtilis cells with different lengths: $1-5 \mu \mathrm{m}$ (blue),

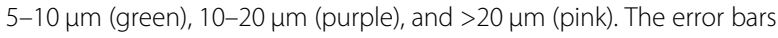
indicate the standard deviations obtained from at least 100 measurements

$c^{1.65}$, at a higher $c$, Wi is larger (see Eq. (2)), and the walldirected interfacial elastic lift force is more dominant ( $\mathrm{El}$ is larger, see Eq. (3)), resulting in more cells trapped at the interface. For longer cells that are located fully in the viscoelastic fluid, the applied elastic lift forces them toward the channel centerline. We noted that the separation performance is more sensitive to PEO concentrations than flow rates. These results agree with previous findings by Tian et al. ${ }^{36}$, in which a co-flow of viscoelastic and Newtonian fluids was used to separate 1 and $2 \mu \mathrm{m}$ particles.

It is worth noting that we also tested the separation performance of the device at a relatively low PEO concentration (i.e., $50 \mathrm{ppm}$ ). We found that a portion of short B. subtilis cells (i.e., $1-5 \mu \mathrm{m}$ ) were directed away from the wall and that the lateral positions of the longer cells were 
closer to the channel wall (see Fig. S7), leading to a reduced decrease in the distance between the lateral positions of the two cell groups (i.e., $<5 \mu \mathrm{m}$ and $>5 \mu \mathrm{m}$ ) compared to the results when $100 \mathrm{ppm}$ PEO was used.

\section{Effects of channel length}

We also investigated the effects of channel length on the lateral positions of $B$. subtilis cells with different morphologies. Here, we used the microchannels with three different lengths, 10, 15, and $20 \mathrm{~mm}$, while the width and height of the channel were fixed at 20 and $50 \mu \mathrm{m}$, respectively. The sample flow and sheath flow (containing $100 \mathrm{ppm}$ PEO in PBS) were fixed at the flow rates of $5 \mu \mathrm{L} /$ $\min$ and $40 \mu \mathrm{L} / \mathrm{min}$, respectively. The corresponding Re was 24.91, Wi was 9.23, and El was 0.37. Figure 5

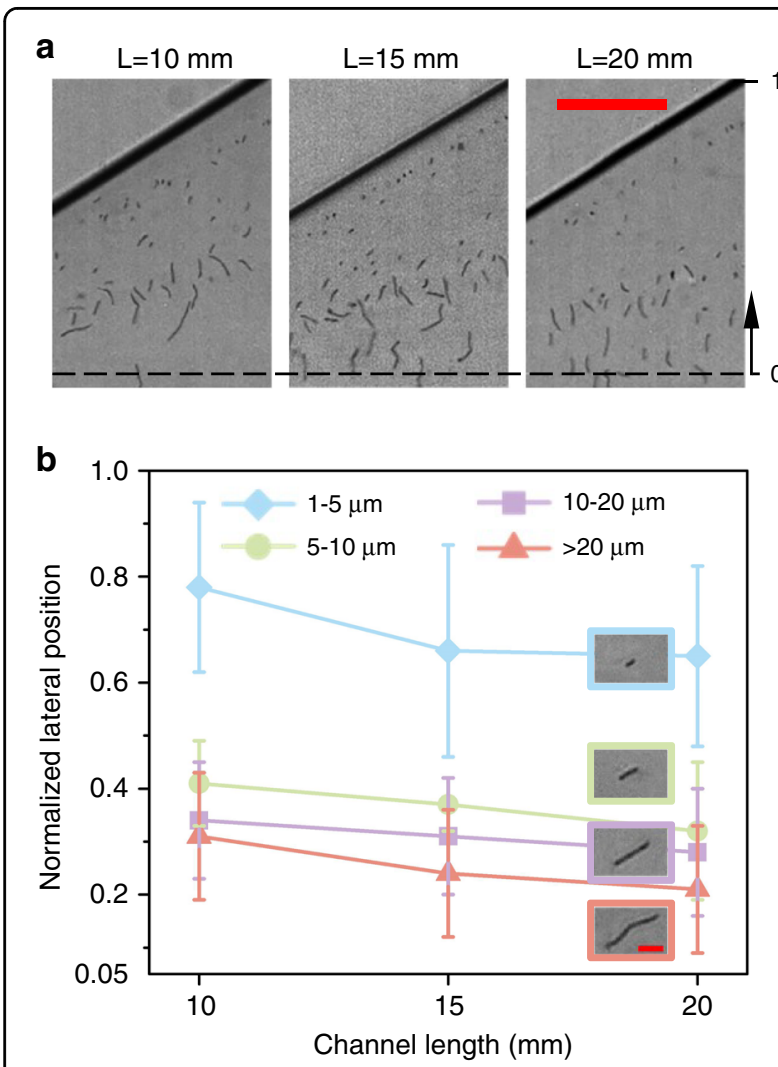

Fig. 5 Effects of channel length on the separation of $B$. subtilis cells using a $100 \mathrm{ppm}$ PEO solution within a rectangular microchannel with three different lengths, 10, 15, and $20 \mathrm{~mm}$. The flow rates of the sample and sheath were $5 \mu \mathrm{L} / \mathrm{min}$ and $40 \mu \mathrm{L} /$ min, respectively. The Re and Wi values were 24.91 and 9.23,

respectively. a Experimental images of $B$. subtilis cells with different lengths at the expansion region of the channels with three different lengths. The black dashed lines represent the channel centerlines. The scale bar represents $50 \mu \mathrm{m}$. b Plots of the average normalized lateral positions for the four groups of $B$. subtilis cells with different lengths:

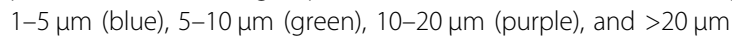
(pink). The error bars indicate the standard deviations obtained from at least 100 measurements compares the normalized lateral positions of the four groups of $B$. subtilis cells at the expansion region (i.e., channel width of $\sim 260 \mu \mathrm{m}, 200 \mu \mathrm{m}$ downstream of the end of the straight channel). The results showed that longer B. subtilis cells had lateral positions closer to the channel centerline than shorter B. subtilis cells in all three channels of different lengths. This is because of the celllength-dependent penetration of the interface between the viscoelastic and Newtonian fluids. Additionally, all four groups of $B$. subtilis cells migrated slightly toward the channel centerline with increasing channel length. This indicates that the combined effects of elastic and inertial lift forces on the length-dependent lateral displacement of B. subtilis cells are cumulative, which agrees with our previous study ${ }^{26}$ that cells of different sizes migrated toward the centerline when the microchannel length increased from $10 \mathrm{~mm}$ to $20 \mathrm{~mm}$. Therefore, we need to carefully select the optimum channel length to maximize the distance between the lateral positions of the cells with different lengths.

\section{Length-based separation of $B$. subtilis cells at the outlets}

Based on the differences in the lateral positions of $B$. subtilis cells that are dependent on cell length, we achieved the label-free, continuous separation of $B$. subtilis cells by length at the outlets. We injected cell-loaded sample fluid and sheath fluid (containing $100 \mathrm{ppm}$ PEO in PBS) into a $15 \mathrm{~mm}$ long microchannel at flow rates of $5 \mu \mathrm{L} / \mathrm{min}$ and $40 \mu \mathrm{L} / \mathrm{min}$, respectively $(\mathrm{Re}=24.91, \mathrm{Wi}=$ $9.23, \mathrm{El}=0.37)$. We designed a serpentine microchannel as a fluidic resistor for each outlet (see Fig. 6a), aiming to reduce the flow rate distortion caused by any small variation in the fluidic resistance at the outlets (e.g., tubing length variation and clogging from debris $)^{21}$. B. subtilis cells with different lengths (i.e., in the range of $1.2-24.6 \mu \mathrm{m}$ ) at the inlets were able to migrate toward and be collected at the different outlets (see Fig. 6b, c).

Shorter B. subtilis cells were found to mainly exit through side outlet 1 , while longer $B$. subtilis cells were mainly collected from middle outlet 4 at the channel centerline. This length-based separation of B. subtilis cells with different lengths at the outlets can also be visualized in Supplementary Movies S2 and S3.

To quantify the efficiency of the length-based separation of B. subtilis cells, we used three parameters ${ }^{26}$ : extraction purity (EP), extraction yield (EY), and enrichment factor (EF) (see Fig. 6d-f). Shorter B. subtilis cells with a length of 1-5 $\mu \mathrm{m}$ (blue) were mainly collected from outlet 1 (O1), with an EP of 97.5\% (Fig. 6d) and an EY of 61.3\% (Fig. 6e). The EP of shorter B. subtilis cells at O3 was $3.8 \%$. This presence of shorter B. subtilis cells at $\mathrm{O} 3$ might be caused by cell adhesion during sample injection and separation, cell-to-cell interactions, or experimental uncertainties. Longer B. subtilis cells with a length of $>5 \mu \mathrm{m}$ (yellow) 
a

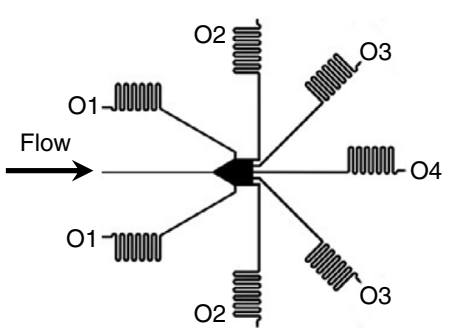

C

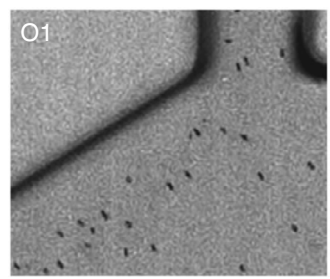

d

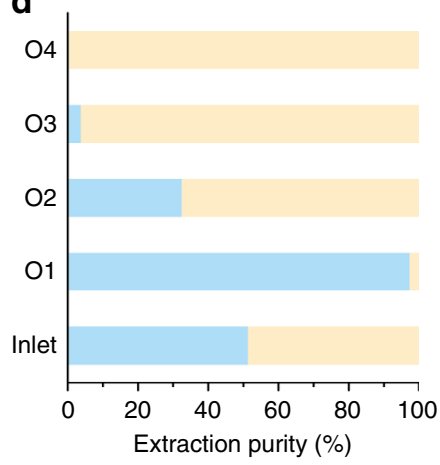

b
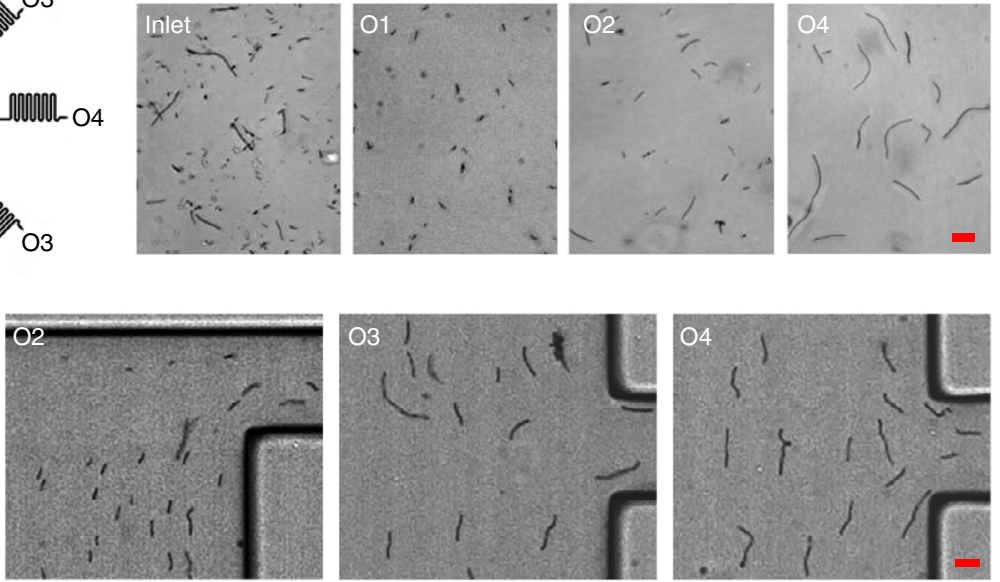

e 80

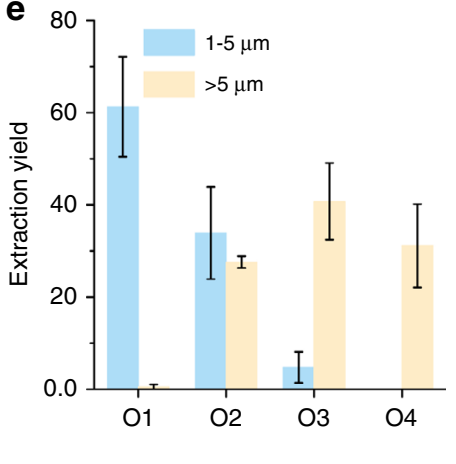

f

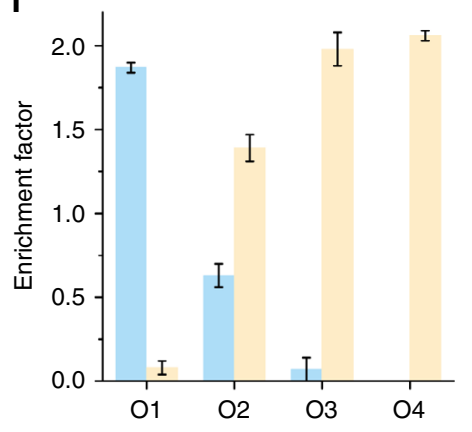

Fig. 6 Separation and enrichment of $B$. subtilis cells with different lengths at the outlets using $100 \mathrm{ppm}$ PEO solution in a $15 \mathrm{~mm}$ long rectangular microchannel. The flow rates of sample and sheath fluids were $5 \mu \mathrm{L} / \mathrm{min}$ and $40 \mu \mathrm{L} / \mathrm{min}$, respectively. a An illustration of the seven outlets for collecting B. subtilis cells of different lengths. $\mathbf{b}$ Snapshot images comparing the proportion of $B$. subtilis cells at the inlet and outlets. c Superimposed experimental images showing that B. subtilis with different lengths are more likely to exit from different outlets: short and long cells are more likely to exit from outlets closer to the sidewall $(\mathrm{O} 1)$ and centerline $(\mathrm{O} 4)$, respectively. Scale bar represents $10 \mu \mathrm{m}$. $\mathbf{d}$ Comparison of the EP for the two groups of $B$. subtilis cells: $1-5 \mu \mathrm{m}$ (blue) and $>5 \mu \mathrm{m}$ (yellow) at the inlet and each outlet. e, $\mathbf{f}$ Bar graphs of (e) EY and (f) EF for the two groups of $B$. subtilis cells with different lengths for each outlet. The error bars represent the standard deviations of three measurements

were found to have relatively high EPs of $96.19 \%$ and $100 \%$ from outlet $3(\mathrm{O} 3)$ and outlet $4(\mathrm{O} 4)$, respectively (see Fig. 6d). This yielded EFs of 1.97 and 2.06 at $\mathrm{O} 3$ and $\mathrm{O} 4$, respectively, for $B$. subtilis cells with a length of $>5 \mu \mathrm{m}$ (see Fig. 6f). Additionally, the separation of the four groups of B. subtilis cells (i.e., $1-5 \mu \mathrm{m}, 5-10 \mu \mathrm{m}, 10-20 \mu \mathrm{m}$, and $>20 \mu \mathrm{m}$ ) was analyzed (see Supplementary Information Fig. S8). We also examined the separation efficiency in terms of EP under different experimental conditions (see Supplementary Information Figure S9), which resulted in a lower EP for the shorter B. subtilis cells (i.e., $1-5 \mu \mathrm{m}$ ) at outlet 1 with higher PEO concentrations. This is because longer cells tended to move toward the channel wall when $c$ was increased (see Fig. 4). Moreover, a portion of shorter B. subtilis cells were found to exist from outlet 4 when the sample flow rate was increased to $50 \mu \mathrm{L} / \mathrm{min}$, as they appeared to migrate toward the channel center under these conditions (see Fig. S6).
Our approach allowed for the successful separation of B. subtilis cells into two distinct groups (cells $<5 \mu \mathrm{m}$ and cells $>5 \mu \mathrm{m}$ ), distinguishing cells in exponential and in stationary growth phases ${ }^{43}$. B. subtilis cells have distinct sizes according to their growth phase. They are approximately $2.7 \mu \mathrm{m}$ long during the stationary phase ${ }^{43}$ and significantly longer during the exponential phase, where they can form chains of filaments ${ }^{44}$. In addition to different phases of growth, the morphology and size variations of B. subtilis are also affected by the growth conditions (e.g., nutrient composition ${ }^{45}$ and antibiotics ${ }^{46,47}$ ) in which $B$. subtilis is cultured.

The geometry of the device and the properties of viscoelastic fluids can be optimized to improve separation efficiency and throughput. For example, specific channel designs can enhance the efficiency of cell separation, including sudden expansion ${ }^{21,48}$, asymmetrical arranged outlet branches and fluidic resistors ${ }^{21,49,50}$ and a cascade 
of microchannels ${ }^{51}$. In addition, some biological polymers, e.g., $\lambda$-DNA, hyaluronic acid, xanthan gum, and extracellular molecules, can be used as alternatives to synthetic polymers (i.e., PEO) in non-Newtonian fluids for particle manipulation ${ }^{37,52}$.

\section{Methods and materials \\ Device fabrication}

The design of the microfluidic device consists of four main elements: two inlets for separately introducing the viscoelastic sheath and sample fluids, a straight rectangular channel with a width of $20 \mu \mathrm{m}$ and three different lengths $(10,15$, and $20 \mathrm{~mm}$ ), an expansion region (with an opening angle of $60^{\circ}$, a total length of $0.9 \mathrm{~mm}$, and a maximum width of $0.6 \mathrm{~mm}$ ), and seven outlets with customized fluidic resistors. The microchannel is uniform with a height of $50 \mu \mathrm{m}$. The master mold made of the negative photoresist SU-8 2050 (MicroChem, Newton, MA, USA) was patterned on a silicon substrate using a laser direct writer (MicroWriter ML3, Durham Magneto Optics, Durham, UK). Degassed poly(dimethyl siloxane) (PDMS; Sylgard 184, Dow Corning, Midland, MI, USA) in liquid form was prepared by mixing the PDMS base with the curing agent in a weight ratio of 10:1, casting over the SU-8 mold, and baking in an oven at $60^{\circ} \mathrm{C}$ for at least $2 \mathrm{~h}$. The PDMS slab containing a negative replica of the microchannels was then peeled off of the mold, and the openings for the inlets and outlets were punched. The PDMS slab and a glass substrate were treated with a plasma cleaner (PX 250, March Instruments, Concord, CA, USA) and assembled. To enhance bonding, the assembled microdevice was placed on a hotplate at $95^{\circ} \mathrm{C}$ for $2 \mathrm{~min}$.

\section{Viscoelastic fluid preparation}

The viscoelastic sheath fluids used in the experiment, PEO solutions with three different concentrations (100, 500 , and $1000 \mathrm{ppm})$, were prepared by dissolving PEO powder $\left(M_{\mathrm{w}}=600 \mathrm{kDa}, 182028\right.$, Sigma-Aldrich, St. Louis, MO, USA) in deionized (DI) water. To completely dissolve the PEO powder, short vortexing $(\sim 10 \mathrm{~s})$ followed by overnight gentle shaking at a speed of $100 \mathrm{rpm} /$ min using an orbital shaker (CLS6791, Corning, Corning, NY, USA) was performed. The properties of the prepared PEO solutions are listed in the Supplementary Information (see Table S3), which were calculated as mentioned in our previous work ${ }^{26}$. The effective relaxation times of the prepared PEO solutions $\lambda$ were estimated from previous times measured by capillary breakup extension rheometry $(\mathrm{CaBER})^{53}$. $\lambda$ can be defined as $\lambda=$ $18 \lambda_{z}\left(c / c^{*}\right)^{0.65}$, where $c$ is the polymer concentration and $c^{*}=0.77 /[\eta]$ is the polymer overlap concentration (the intrinsic viscosity $\left.[\eta]=0.072 M_{W}^{0.65}\right)^{54} . \quad \lambda_{z}=$ $f[\eta]\left(M_{w}\right) \eta_{s} / R T$ is the relaxation time predicted by Zimm theory ${ }^{54,55}$, where $f(=0.463)$ is the prefactor dependent on solvent quality, $\eta_{s}\left(=1 \times 10^{-3} \mathrm{~Pa} \cdot \mathrm{s}\right)$ is the solvent viscosity, $R\left(=8.314 \mathrm{~J} \mathrm{~mol}^{-1} \mathrm{~K}\right)$ is the gas constant, and $T$ $(=293 \mathrm{~K})$ is the absolute temperature. Since the polymeric contribution to the solution viscosity $\eta_{p}$ can be expressed as $[\eta] c \eta_{s}=0.072 c \eta_{s} M_{W}^{0.65}$, the dynamic viscosity $\eta=\eta_{s}+0.072 c \eta_{s} M_{W}^{0.65}$.

\section{Analysis of separation performance}

The separation efficiency can be characterized by three parameters $^{56}$. The EP considers the proportion of the number of a cell group $a$ in outlet $i$ relative to the total number of cells extracted from this outlet; EP reports the cell composition of a given outlet. EY is calculated as the number of cell groups $a$ extracted from outlet $i$ over the total number of this cell group injected from the inlet; EY is the outlet collection efficiency of a given cell group. EF is defined as the proportion of cells $a$ in outlet $i$ to the proportion of cells at the inlet.

$$
\begin{aligned}
& E P=\frac{N_{a}\left(\text { outlet }_{i}\right)}{N_{\text {total }}\left(\text { outlet }_{i}\right)}, E Y=\frac{N_{a}\left(\text { outlet }_{i}\right)}{N_{a}(\text { inlet })}, \\
& E F=\frac{N_{a}\left(\text { outlet }_{i}\right) / N_{\text {total }}\left(\text { outlet }_{i}\right)}{N_{a}(\text { inlet }) / N_{\text {total }}(\text { inlet })} .
\end{aligned}
$$

\section{Cell preparation}

B. subtilis ATCC 6633 cells were grown overnight at $37^{\circ} \mathrm{C}$ with shaking at $200 \mathrm{rpm}$ in $5 \mathrm{~mL}$ of Luria-Bertani broth (51208, Sigma-Aldrich, St. Louis, MO, USA). Overnight cultures were spun down at $5000 \mathrm{~g}$ at $4{ }^{\circ} \mathrm{C}$ for $5 \mathrm{~min}$ and washed with $5 \mathrm{~mL}$ of ice-cold $1 \times$ phosphatebuffered saline (PBS; 10010023, Thermo Fisher Scientific, Waltham, MA). This step was repeated once for a total of two washes. To characterize the trajectories of the fixed $B$. subtilis cells in the viscoelastic microfluidic device, the washed cell suspensions were added to $4.5 \mathrm{~mL}$ of ice-cold $70 \%$ ethanol while vortexing gently. Cells were then incubated at $4{ }^{\circ} \mathrm{C}$ for $2 \mathrm{~h}$ followed by centrifugation at $1000 \mathrm{~g}$ for $5 \mathrm{~min}$. The supernatant was carefully removed, and the cells were resuspended in $1 \times \mathrm{PBS}$ and incubated at ambient temperature for $15 \mathrm{~min}$. These fixed cells were used for the cell separation experiment in this study, as they can resist lysis for a long time at $4{ }^{\circ} \mathrm{C}$ in a refrigerator. Then, $25 \mu \mathrm{L}$ of methylene blue (50484, Sigma-Aldrich, St. Louis, MO, USA) was added to $500 \mu \mathrm{L}$ of final solution with a cell concentration of $2 \times 10^{7} \mathrm{cell} / \mathrm{mL}$ and cocultured for $20 \mathrm{~min}$ for downstream experiments.

\section{Experimental setup}

Before the experimental operation, the microdevices were prefilled with $0.1 \%$ Tween $^{\circledR} 20$ (P9416, Sigma-Aldrich, St. Louis, MO, USA) supplemented with 
deionized (DI) water to prevent the cells from adhering to the channel walls. Afterwards, B. subtilis cell suspensions (sample fluid) and PEO solution (sheath fluid) were simultaneously injected into the microchannel via two separate inlets using two syringe pumps (Fusion 100, Chemyx, Stafford, TX, USA). The trajectories of the $B$. subtilis cells were monitored and recorded with a highspeed camera (Phantom Miro LC320S, Vision Research, Wayne, NJ, USA) mounted on an inverted microscope (Eclipse Ti-U, Nikon, Tokyo, Japan) at a fixed exposure time of $30 \mu \mathrm{s}$. Recorded videos and images were postprocessed and analyzed by ImageJ software (National Institutes of Health (NIH), Bethesda, MD, USA). The lateral position distributions of B. subtilis cells were calculated based on the distance between the centroids of the cells and the channel centerline using stacked images captured $100 \mu \mathrm{m}$ downstream of the end of the straight channel. The lengths of the cells were determined by measuring the Feret diameters of the cells. Sorted cells were collected at the outlets and counted to calculate separation efficiency in terms of EP, EY, and EF.

\section{Conclusion}

In this work, we demonstrated the label-free continuous separation of B. subtilis cells by length using a co-flow of viscoelastic and Newtonian fluids in a straight rectangular microchannel. B. subtilis cells varying in morphology were found to have different lateral equilibrium positions, dependent on cell length due to elasto-inertial effects and separation. The results revealed that these factors generally have a greater effect on the migration of shorter cells (i.e., $<5 \mu \mathrm{m}$ ) than that of longer cells (i.e., $>5 \mu \mathrm{m}$ ). B. Subtills cell separation did not increase monotonically with PEO concentration because of the combined elastic and inertial effects, but microchannel length strongly influenced cell migration because the combined effects of elastic and inertial lift forces are cumulative. We performed a systematic experimental study of the influences of the flow rate ratio, PEO concentration, and channel length on the lateral positions of B. subtilis cells of different lengths. Moreover, length-based separation and enrichment of B. subtilis cells were achieved at the outlets. Shorter cells were found to have a high EP of $97.5 \%$ at the outlet closest to the channel wall, and longer cells were mainly collected from the outlet in the channel center with an EY of $65.2 \%$ and an EF of up to 6.88. Our study is the first to demonstrate that viscoelastic microfluidics can be adopted for the separation of bacterial cells by length, an important biomarker. To date, few theoretical models have been developed to predict particle migration in Newtonian and viscoelastic fluidics coflow systems. However, several computational fluid dynamics simulations ${ }^{28,57}$ and novel imaging instruments $^{52,58}$ (e.g., digital holography) have been adopted to investigate the velocity profiles and particle focusing patterns. These approaches can potentially facilitate detailed investigations to elucidate the underlying mechanisms. The current platform is expected to be used as a powerful tool for basic studies and industrial applications with bacterial cells by integrating functional components (e.g., focusing and imaging $)^{59,60}$, functional genomics, and metabolic and genetic engineering techniques.

\section{Acknowledgements \\ This work was supported by an Australian Research Council (ARC) Discovery Project Grant (DP200102269) to ML and AKC. PL acknowledges support from the Overseas Research Program for Outstanding Young and Middle-aged College Students and Principals of Jiangsu Province, the Scientific Research and Innovation Team of Suqian University (2021td07), and the Suqian Science \& Technology Project (Z2020136). AKC was supported by an Australian Research Council (ARC) DECRA fellowship (DE180100929). HL was supported by International Macquarie University Research Excellence Scholarships (iMQRES) for graduate study. DY was supported by an Alfred Deakin Postdoctoral Research Fellowship from Deakin University.}

\section{Author details}

${ }^{1}$ Suqian University, Suqian 223800, China. ${ }^{2}$ School of Engineering, Macquarie University, Sydney, NSW 2109, Australia. ${ }^{3}$ Department of Physics and Astronomy, Macquarie University, Sydney, NSW 2109, Australia. ${ }^{4}$ ARC Centre of Excellence in Synthetic Biology, Department of Molecular Science, Macquarie University, Sydney, NSW 2109, Australia. ${ }^{5}$ Centre for Regional and Rural Futures,

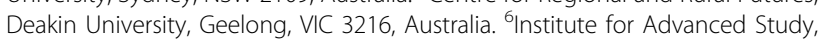
Shenzhen University, Shenzhen 518060, China. ${ }^{7}$ Biomolecular Discovery

Research Centre, Macquarie University, Sydney, NSW 2109, Australia

\section{Author contributions}

P.L. and H.L. contributed equally to this work. M.L. and A.K.C. conceived the study idea. H.L. and P.L. designed the experiments. P.L. and H.L. performed the experiments. L.S. prepared the cells. P.L., H.L., D.Y., and S.Y. analyzed the data. M.L. supervised the project. All authors contributed to paper writing.

\section{Conflict of interest}

The authors declare no competing interests.

Supplementary information The online version contains supplementary material available at https://doi.org/10.1038/s41378-021-00333-3.

Received: 13 July 2021 Revised: 8 November 2021 Accepted: 9 November 2021

Published online: 19 January 2022

\section{References}

1. Su, Y., Liu, C., Fang, H. \& Zhang, D. Bacillus subtilis: A universal cell factory for industry, agriculture, biomaterials and medicine. Microb. Cell Fact. 19, 173 (2020).

2. Errington, J. \& Aart, L. T. V. D. Microbe Profile: Bacillus subtilis: Model organism for cellular development, and industrial workhorse. Microbiology 166, 425-427 (2020).

3. Harwood, C. R. Bacillus subtilis and its relatives: Molecular biological and industrial workhorses. Trends Biotechnol. 10, 247-256 (1992).

4. Lopez, D., Vlamakis, H. \& Kolter, R. Generation of multiple cell types in Bacillus subtilis. FEMS Microbiol. Rev. 33, 152-163 (2009).

5. Nordholt, N., van Heerden, J. H. \& Bruggeman, F. J. Biphasic cell-size and growth-rate homeostasis by single Bacillus subtilis cells. Curr. Biol. 30, 2238-2247.e2235 (2020)

6. Liu, H. et al. Microdroplet enabled cultivation of single yeast cells correlates with bulk growth and reveals subpopulation phenomena. Biotechnol. Bioeng. 118, 647-658 (2021).

7. Kearns, D. B. \& Losick, R. Cell population heterogeneity during growth of Bacillus subtilis. Genes Dev. 19, 3083-3094 (2005). 
8. Nishihara, T. \& Freese, E. Motility of Bacillus subtilis during growth and sporulation. J. Bacteriol. 123, 366-371 (1975)

9. Jones, L. J. F., Carballido-López, R. \& Errington, J. Control of Cell Shape in Bacteria: Helical, actin-like filaments in Bacillus subtilis. Cell 104, 913-922 (2001)

10. Russell, A. D. Lethal effects of heat on bacterial physiology and structure. Sci. Prog. 86, 115-137 (2003).

11. Tasaki, S., Nakayama, M. \& Shoji, W. Morphologies of Bacillus subtilis communities responding to environmental variation. Dev. Growth Differ. 59, 369-378 (2017).

12. Sassine, J., Sousa, J., Lalk, M., Daniel, R. A. \& Vollmer, W. Cell morphology maintenance in Bacillus subtilis through balanced peptidoglycan synthesis and hydrolysis. Sci. Rep. 10, 17910 (2020).

13. Justice, S. S., Hunstad, D. A., Cegelski, L. \& Hultgren, S. J. Morphological plasticity as a bacterial survival strategy. Nat. Rev. Microbiol. 6, 162-168 (2008).

14. Morijiri, T., Yamada, M., Hikida, T. \& Seki, M. Microfluidic counterflow centrifugal elutriation system for sedimentation-based cell separation. Microfluid. Nanofluidics 14, 1049-1057 (2013).

15. Oh, M.-J., Kuhr, F., Byfield, F. \& Levitan, I. Micropipette aspiration of substrateattached cells to estimate cell stiffness. J. Vis. Exp. 3886, https://doi.org/ 10.3791/3886 (2012).

16. Behdani, B. et al. Shape-based separation of micro-/nanoparticles in liquid phases. Biomicrofluidics 12, $051503-051503$ (2018).

17. Valero, A. et al. Tracking and synchronization of the yeast cell cycle using dielectrophoretic opacity. Lab Chip 11, 1754-1760 (2011).

18. Zhou, R., Bai, F. \& Wang, C. Magnetic separation of microparticles by shape. Lab Chip 17, 401-406 (2017).

19. Beech, J. P., Holm, S. H., Adolfsson, K. \& Tegenfeldt, J. O. Sorting cells by size, shape and deformability. Lab Chip 12, 1048-1051 (2012).

20. Sugaya, S., Yamada, M. \& Seki, M. Observation of nonspherical particle behaviors for continuous shape-based separation using hydrodynamic filtration. Biomicrofluidics 5, 24103-24103 (2011).

21. Li, M., Muñoz, H. E., Goda, K. \& Di Carlo, D. Shape-based separation of microalga Euglena gracilis using inertial microfluidics. Sci. Rep. 7, 10802 (2017)

22. Li, M. et al. Inertial focusing of ellipsoidal Euglena gracilis cells in a stepped microchannel. Lab Chip 16, 4458-4465 (2016).

23. Beech, J. P. et al. Separation of pathogenic bacteria by chain length. Anal. Chim. Acta 1000, 223-231 (2018).

24. Ranjan, S., Zeming, K. K., Jureen, R., Fisher, D. \& Zhang, Y. DLD pillar shape design for efficient separation of spherical and non-spherical bioparticles. Lab Chip 14, 4250-4262 (2014).

25. Condina, M. R. et al. Rapid separation and identification of beer spoilage bacteria by inertial microfluidics and MALDI-TOF mass spectrometry. Lab Chip 19, 1961-1970 (2019).

26. Liu, P. et al. Separation and enrichment of yeast saccharomyces cerevisiae by shape using viscoelastic microfluidics. Anal. Chem. 93, 1586-1595 (2021).

27. Mutlu, B. R., Edd, J. F. \& Toner, M. Oscillatory inertial focusing in infinite microchannels. Proc. Nat. I Acad. Sci. USA 115, 7682 (2018).

28. Zhou, J. \& Papautsky, I. Viscoelastic microfluidics: Progress and challenges. Microsyst. Nanoeng. 6, 113 (2020).

29. Cruz, J., Graells, T., Walldén, M. \& Hjort, K. Inertial focusing with sub-micron resolution for separation of bacteria. Lab Chip 19, 1257-1266 (2019).

30. Pitt, W. G. et al. Rapid separation of bacteria from blood-review and outlook. Biotechnol. Prog. 32, 823-839 (2016).

31. Chung, A. J. A minireview on inertial microfluidics fundamentals: Inertial particle focusing and secondary flow. Biochip J. 13, 53-63 (2019).

32. Faridi, M. A. et al. Elasto-inertial microfluidics for bacteria separation from whole blood for sepsis diagnostics. J. Nanobiotechnol. 15, 3 (2017).

33. Nam, J., Lim, H., Kim, D., Jung, H. \& Shin, S. Continuous separation of microparticles in a microfluidic channel via the elasto-inertial effect of nonNewtonian fluid. Lab Chip 12, 1347-1354 (2012).

34. Tian, F. et al. Label-free isolation of rare tumor cells from untreated whole blood by interfacial viscoelastic microfluidics. Lab Chip 18, 3436-3445 (2018).
35. Zhou, J. et al. Isolation of circulating tumor cells in non-small-cell-lung-cancer patients using a multi-flow microfluidic channel. Microsyst. Nanoeng. 5, 8 (2019).

36. Tian, F. et al. Microfluidic co-flow of Newtonian and viscoelastic fluids for highresolution separation of microparticles. Lab Chip 17, 3078-3085 (2017).

37. Liu, C. et al. Field-free isolation of exosomes from extracellular vesicles by microfluidic viscoelastic flows. ACS Nano. 11, 6968-6976 (2017).

38. Yuan, D. et al. Sheathless separation of cyanobacterial anabaena by shape using viscoelastic microfluidics. Anal. Chem. 93, 12648-12654 (2021).

39. Di Carlo, D., Edd, J. F., Humphry, K. J., Stone, H. A. \& Toner, M. Particle segregation and dynamics in confined flows.Phys. Rev. Lett. 102, 094503 (2009).

40. Yang, S., Kim, J. Y., Lee, S. J., Lee, S. S. \& Kim, J. M. Sheathless elasto-inertial particle focusing and continuous separation in a straight rectangular microchannel. Lab Chip 11, 266-273 (2011).

41. Pathak, J. A., Ross, D. \& Migler, K. B. Elastic flow instability, curved streamlines, and mixing in microfluidic flows. Phys. Fluids 16, 4028-4034 (2004).

42. Lu, X. \& Xuan, X. Elasto-inertial pinched flow fractionation for continuous shape-based particle separation. Anal. Chem. 87, 11523-11530 (2015).

43. Li, K. et al. Atomic force microscopy of side wall and septa peptidoglycan from Bacillus subtilis reveals an architectural remodeling during growth. Front Microbiol. 9, 620 (2018).

44. Lee, S., Wu, L. J. \& Errington, J. Microfluidic time-lapse analysis and reevaluation of the Bacillus subtilis cell cycle. MicrobiologyOpen 8, e876 (2019).

45. Sauls John, T. et al. Control of Bacillus subtilis replication initiation during physiological transitions and perturbations. mBio 10, e02205-e02219 (2019).

46. Hyde, A. J., Parisot, J., McNichol, A. \& Bonev, B. B. Nisin-induced changes in Bacillus morphology suggest a paradigm of antibiotic action. Proc. Nat. I Acad. Sci. USA 103, 19896 (2006).

47. McAuley, S. et al. A chemical inhibitor of cell growth reduces cell size in Bacillus subtilis. ACS Chem. Biol. 14, 688-695 (2019).

48. Nguyen, N. et al. Inertial Microfluidics with Integrated Vortex Generators Using Liquid Metal Droplets as Fugitive Ink. Adv. Funct. Mater. https://doi.org/ 10.1002/adfm.201901998 (2019).

49. Di Carlo, D. Inertial microfluidics. Lab Chip 9, 3038-3046 (2009).

50. Takagi, J., Yamada, M., Yasuda, M. \& Seki, M. Continuous particle separation in a microchannel having asymmetrically arranged multiple branches. Lab Chip $\mathbf{5}$, 778-784 (2005).

51. Wei Hou, H. et al. A microfluidics approach towards high-throughput pathogen removal from blood using margination. Biomicrofluidics $\mathbf{6}, 024115$ (2012).

52. Zhou, Z. et al. Inertial microfluidics for high-throughput cell analysis and detection: A review. Analyst https://doi.org/10.1039/D1AN00983D (2021).

53. Tirtaatmadja, V., McKinley, G. H. \& Cooper-White, J. J. Drop formation and breakup of low viscosity elastic fluids: Effects of molecular weight and concentration. Phys. Fluids. 18, 043101 (2006).

54. Rodd, L. E., Scott, T. P., Boger, D. V., Cooper-White, J. J. \& McKinley, G. H. The inertio-elastic planar entry flow of low-viscosity elastic fluids in microfabricated geometries. J. Non-Newton. Fluid Mech. 129, 1-22 (2005).

55. Rodd, L. E., Cooper-White, J. J., Boger, D. V. \& McKinley, G. H. Role of the elasticity number in the entry flow of dilute polymer solutions in microfabricated contraction geometries. J. Non-Newton. Fluid Mech. 143, 170-191 (2007).

56. Masaeli, M. et al. Continuous inertial focusing and separation of particles by shape. Phys. Rev. X 2, 031017 (2012).

57. Tang, W. et al. Channel innovations for inertial microfluidics. Lab Chip 20, 3485-3502 (2020).

58. Hochstetter, A. Lab-on-a-Chip Technologies for the Single Cell Level: Separation, Analysis, and Diagnostics. Micromachines. 11, https://doi.org/10.3390/ mi11050468 (2020).

59. Zhang, T. et al. Focusing of sub-micrometer particles in microfluidic devices. Lab Chip 20, 35-53 (2020).

60. Nitta, N. et al. Intelligent image-activated cell sorting. Cell 175, 266-276.e213 (2018). 